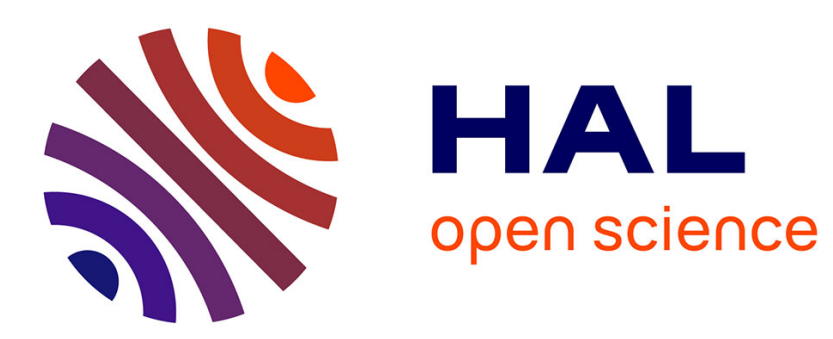

\title{
Safety Margins Confidence Estimation for a Passive Residual Heat Removal System
}

Enrico Zio, Francesco Di Maio, J. Tong

\section{To cite this version:}

Enrico Zio, Francesco Di Maio, J. Tong. Safety Margins Confidence Estimation for a Passive Residual Heat Removal System. Reliability Engineering and System Safety, 2010, 95 (8), pp.828-836. 10.1016/j.ress.2010.03.006 . hal-00610473

\section{HAL Id: hal-00610473}

https://hal-centralesupelec.archives-ouvertes.fr/hal-00610473

Submitted on 26 Jul 2012

HAL is a multi-disciplinary open access archive for the deposit and dissemination of scientific research documents, whether they are published or not. The documents may come from teaching and research institutions in France or abroad, or from public or private research centers.
L'archive ouverte pluridisciplinaire HAL, est destinée au dépôt et à la diffusion de documents scientifiques de niveau recherche, publiés ou non, émanant des établissements d'enseignement et de recherche français ou étrangers, des laboratoires publics ou privés. 


\title{
SAFETY MARGINS CONFIDENCE ESTIMATION FOR A PASSIVE RESIDUAL HEAT REMOVAL SYSTEM
}

\author{
Enrico Zio ${ }^{1}$, Francesco Di Maio ${ }^{1}$, Jiejuan Tong $^{2}$ \\ ${ }^{1}$ Energy Department, Polytechnic of Milan \\ Via Ponzio 34/3, 20133 Milano, Italy \\ enrico.zio@polimi.it \\ ${ }^{2}$ INET, Institute of Nuclear and New Energy Technology \\ Tsinghua University, Beijing,100084, China
}

\begin{abstract}
For licensing purposes, safety cases of Nuclear Power Plants (NPPs) must be presented at the Regulatory Authority with the necessary confidence on the outcomes of the models used to analyze the plant safety behavior. In the present work, we consider the problem of providing a quantitative indication of the confidence in the safety margin estimation by a model with uncertain inputs giving in output the maximum outlet water temperature of the Residual Heat Removal system (RHRs) in accident scenarios of the High Temperature Reactor-Pebble Modular (HTR-PM). The quantitative evaluation is carried out by means of a computational procedure of literature, based on Order Statistics (OS). The procedure is analyzed with respect to some of its key parameters defining the sample size and the number of uncertain inputs.
\end{abstract}




\section{NOTATION AND LIST OF ACRONYMS}

\begin{tabular}{|c|c|}
\hline OS & Order Statistics \\
\hline $\mathrm{BE}$ & Best-Estimate \\
\hline NPP & Nuclear Power Plant \\
\hline HTR-PM & High Temperature Reactor-Pebble Modular \\
\hline DBA & Design Basis Accident \\
\hline BDBA & Beyond Design Basis Accident \\
\hline RHRs & Residual Heat Removal system \\
\hline BSFs & Basic Safety Functions \\
\hline $\bar{x}$ & Input values vector \\
\hline$x_{m}$ & $m^{\text {th }}$ element of the input vector \\
\hline $\bar{y}$ & Output values vector \\
\hline$T(\bar{x})$ & Function that maps the input vector $\bar{x}$ into the output vector $\bar{y}$ \\
\hline$L_{j}$ & Lower threshold for the $j^{\text {th }}$ output parameter \\
\hline$U_{j}$ & Upper threshold for the $j^{\text {th }}$ output parameter \\
\hline $\bar{x}^{(i)}$ & $i^{\text {th }}$ element of the representative sample of independent input vectors \\
\hline $\bar{y}^{(i)}$ & $i^{\text {th }}$ element of the representative sample of independent output vectors \\
\hline$k$ & Index of the accident scenario \\
\hline$M\left(y_{j}, k\right)$ & Safety margin relative to the $j^{\text {th }}$ safety parameter for the $k^{\text {th }}$ accident scenario \\
\hline$y_{j}(k)$ & $j^{\text {th }}$ safety parameter relative to the $k^{\text {th }}$ accident scenario \\
\hline$N$ & Number of simulations \\
\hline$r$ & Position of the ordered sample of simulations \\
\hline$Y$ & Ordered set of values resulting from running the code $N$ times \\
\hline$\beta$ & Confidence value \\
\hline$\gamma$ & Coverage value \\
\hline$m$ & Number of values that lie beyond the $\gamma$ coverage extent \\
\hline$\gamma y$ & Real $\gamma^{\text {th }}$ percentile \\
\hline$y_{m}$ & Estimated $\gamma^{\text {th }}$ percentile \\
\hline $0.95 y$ & Real $95^{\text {th }}$ percentile \\
\hline $0.95 \hat{y}$ & $95^{\text {th }}$ percentile estimate \\
\hline $0.95 \hat{y}_{0.5}$ & Estimated median of the distribution of ${ }_{0.95} \hat{y}$ \\
\hline $0.95 \hat{y}^{[r]}$ & Lower bound of random interval covering the median of the distribution of ${ }_{0.95} \hat{y}$ \\
\hline $0.95 \hat{y}^{[s]}$ & Upper bound of random interval covering the median of the distribution of ${ }_{0.95} \hat{y}$ \\
\hline
\end{tabular}

\section{INTRODUCTION}

Conservative calculations are traditionally performed for the verification of the safety performance of a Nuclear Power Plant (NPP) under Design Basis Accidents (DBAs) conditions, in terms of the values reached by selected safety parameters in comparison to 
threshold values enforced by regulation to ensure that sufficient safety margins are maintained for the integrity of the defense-in-depth barriers. The differences between the conservatively computed safety parameter values and the thresholds give the so called safety margins. Conservatism is introduced in the calculations to account for the uncertainties in the model representation of the actual plant behavior.

For the Beyond Design Basis Accidents (BDBAs), the conservative approach is being challenged by a more realistic, Best-Estimate (BE) analysis, which sets forth the calculation of safety margins with realistic models and BE assumptions to account for the consequences related to the failures in some protective barriers.

On one side, the reduction in the conservatism of the analyses leads to more efficient plant design and operation. On the other side, the relaxation of the conservatisms entails that sensitivity and uncertainty analyses be carried out to properly quantify the safety margins while capturing the associated uncertainty for confidence evaluation. This requires a revision in probabilistic terms of the concept of safety margins [Gavrilas et al., 2004] and repeated model runs for the associated sensitivity and uncertainty analyses.

This is even more so when $i$ ) analyzing requests for changes to the licensing bases, within a risk-informed decision-making philosophy and ii) checking design solutions of new NPPs whose safety analysis relies on newly developed models and codes, because the combination of the uncertainties in the analysis could reduce significantly and in an unexpected way the safety margins [USNRC, 1998; Martorell et al., 2006]. This situation may increase the risk of accidents, leading to the dissatisfaction of some of the Basic Safety Functions (BSFs) (i.e., reactivity control, residual heat removal, primary pressure 
control and containment release) that have to be carried out by the implemented safety systems to avoid major dangerous consequences.

In general, uncertainty affecting the plant behavior can be considered of two types: that due to inherent variability in the system behavior and that due to lack of knowledge and information on the system. The former type of uncertainty is often referred to as objective, aleatory, stochastic whereas the latter is called subjective, epistemic, state-ofknowledge [Apostolakis, 1990; Helton, 2004].

The distinction between aleatory and epistemic uncertainty plays a particularly important role in the risk assessment framework applied to complex engineered systems. In the context of risk analysis, the aleatory uncertainty is related to the occurrence of the events which define the various possible accident scenarios whereas epistemic uncertainty arises from a lack of knowledge of fixed but poorly known parameter values entering the evaluation of the probabilities and consequences of the accident scenarios.

The present work addresses the epistemic uncertainty affecting the evaluation of the safety margins. Under the probabilistic viewpoint here undertaken to represent uncertainties, the code for safety margin evaluation needs to be repeatedly run with different values of the thermal-hydraulic parameters, sampled from predefined probability distributions; the outcomes of these runs are then statistically analyzed to estimate with a specified confidence a given percentile of the distribution of the safety parameter used to calculate the safety margin [Guba et al., 2003; Nutt et al., 2004]. The confidence intervals of the estimated safety margins is also computed: this additional information provides a realistic refinement of the estimates that is beneficial to power plant owners; on the other hand, from the viewpoint of the regulatory body it increases 
the robustness of the safety case by allowing verification of the fact that uncertainty in the safety margin estimates does not lead to exceedance of the regulatory safety thresholds.

In general terms, because of the large computing times required to run the codes, the procedure can be computationally quite expensive. Thus, tin this work the statistical analyses of the model evaluations for obtaining confidence intervals for safety parameters estimates rely on the use of Order Statistics (OS), along a non-parametric approach initially explored by [Wilks, 1941; Wilks, 1942]; this brings the advantage that the number of code calculations needed for safety margins evaluation is independent of the number of uncertain input parameters and provides a given confidence on the reliability of the calculated point-estimate obtained with a limited number of code runs.

Figure 1 shows a schematic sketch of the non-parametric procedure here adopted [Secchi et al., 2008]; for ease of illustration, a single safety parameter $y$ is considered. By this procedure for safety margin calculation, the analyst can produce results with the level of confidence against uncertainty required for presenting a robust safety case to the Regulatory Authority.

The approach is applied to a case study regarding a set of accident scenarios related to the Residual Heat Removal system (RHRs) of the High Temperature Reactor-Pebble Modular (HTR-PM) [Zhengy et al., 2008]. The application is of safety significance because the RHRs is a passive safety system which operates without external input energy [IAEA, 1991] and is thus expected to contribute significantly to the improvement of plant safety [Mathews et al., 2008]. 
However, the uncertainties involved in the modeling of the behavior of passive systems are usually larger than in active systems, due to lack of data on some underlying phenomena and scarce or null operating experience over the wide range of conditions encountered during operation [Pagani et al., 2005].

This situation may actually increase the risk of accidents leading to the dissatisfaction of some of the BSFs (i.e., reactivity control, residual heat removal, primary pressure control and containment release) that the safety systems are designed for. In fact, deviations in the natural forces and in the conditions of the underlying physical principles from the model expected ones can impair the function of the system itself [Marquès et al., 2005; Burgazzi, 2007].

The paper organization is as follows. In Section 2, the basic principles underpinning the BE nuclear safety analysis in the presence of uncertainties are provided together with an illustration of the method for uncertainty estimation here employed. In Section 3, the main characteristics of the High Temperature Reactor-Pebble Modular (HTR-PM) are briefly introduced, the Residual Heat Removal system (RHRs) accident scenarios are described and the simulations performed to analyze the system response to the accident scenario are presented. In Section 4, the results of the application of the proposed framework for the estimation of the safety margin of the maximum outlet water temperature reached during the accidents described in Section 3 are provided. Finally, some conclusions are drawn in Section 5. 


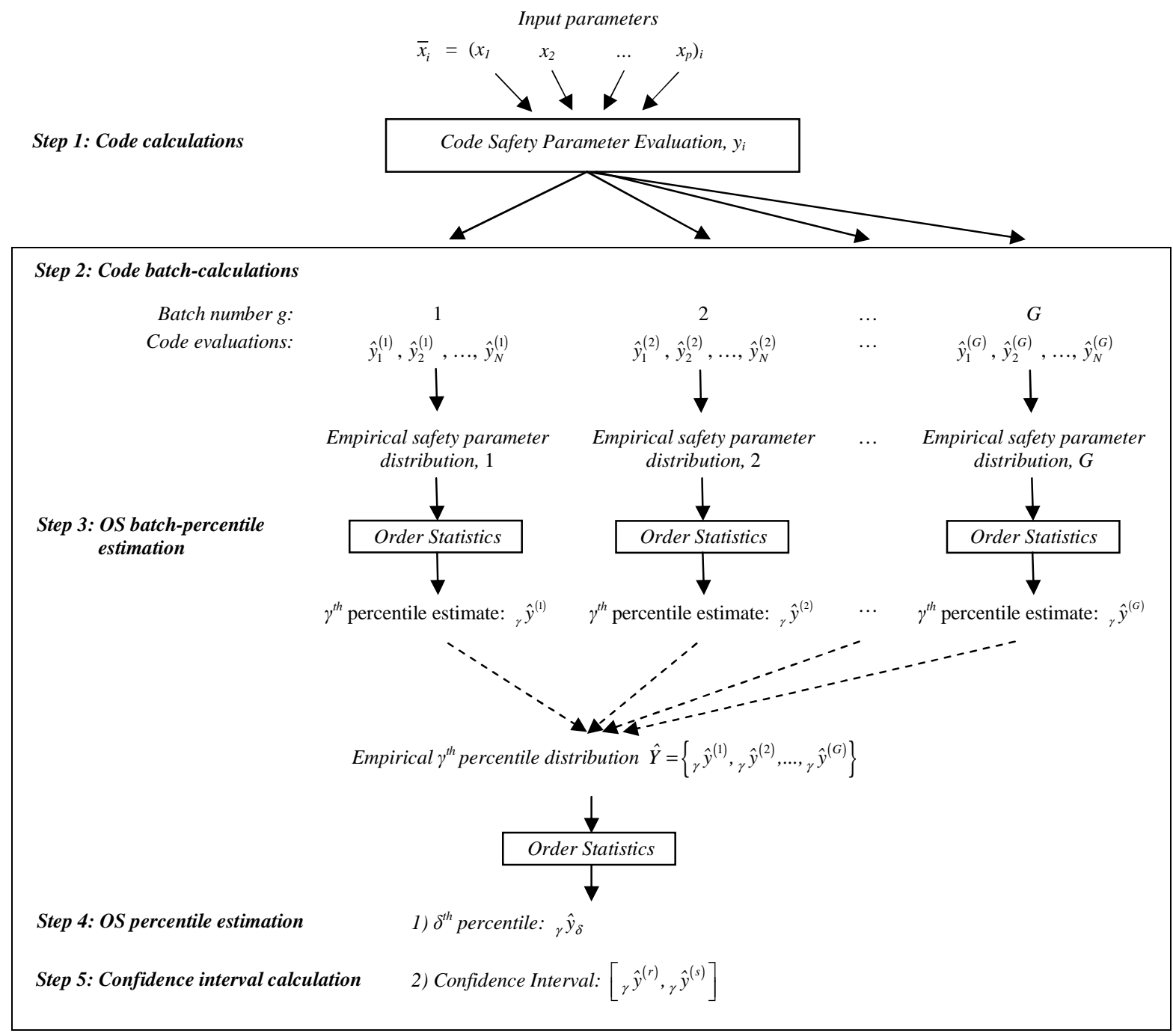

Figure 1 Flowchart of the non-parametric procedure for percentile and confidence interval estimation [Secchi et al., 2008]

\section{SAFETY MARGIN ESTIMATION}

\subsection{The mathematical model}


A quantitative model for safety analysis of a nuclear power plant may be viewed as composed of three main elements: an input vector $\bar{x}=\left\{x_{1}, x_{2}, \ldots, x_{p}\right\}$, a BE simulator code and an output vector $\bar{y}=\left\{y_{1}, y_{2}, \ldots, y_{l}\right\}$. The elements of the input vector $\bar{x}$ are all the model parameters and input variables needed to calculate one realization of the output variables $\bar{y}$ describing the system response. The simulation code can be regarded as a black box which implements the complex, multidimensional, nonlinear function that maps the input vector $\bar{x}$ into the output vector $\bar{y}$ [Guba et al., 2003]:

$$
\bar{y}=T(\bar{x})
$$

For fixed values of $\bar{x}$, the output values $\bar{y}$ is deterministically computed.

\subsection{Formulation of safety margins in presence of uncertainty}

The defense-in-depth principle of nuclear safety is founded on the implementation of protective barriers between the radioactive products and the environment. Each barrier is a physical device whose integrity is measured with reference to given characteristic safety parameters. When a barrier is subjected to abnormal conditions, some of the related safety parameters may exceed their safety envelope, which results in the failure of the barrier.

With reference to a generic accident scenario $k$ and a characteristic safety variable $y_{j}(k)$ to be limited from above by an upper threshold limit $U_{j}$, the safety margin $M\left(y_{j}, k\right)$ can be defined as: 


$$
M\left(y_{j}, k\right)=U_{j}-y_{j}
$$

The dual definition of the safety margin for a safety parameter $y_{j}$ to be limited from below by a threshold value $L_{j}$ is straightforward.

\subsection{Estimation of safety margins}

The safety margins are calculated by running the thermal-hydraulic codes used for safety analysis. In presence of uncertainty, a large number of runs of the code may be required to adequately represent the full distribution of the safety parameter values in output. The problem is that the computer runs of the complex models of plant dynamics used for safety analysis are computationally very expensive. To overcome this hurdle one may resort either to simplified analytical/numerical models, such as those based on lumped effective parameters [Marseguerra et al., 2004], or to empirical models, e.g. artificial neural networks and fuzzy logic systems [Marseguerra et al., 2003], suitably set up so as to best fit to the data available from the plant.

Another possibility to reduce the computational burden, which may be used in combination with the former ones, is to only compute some percentiles of the output distribution, estimated with a limited number of runs. In this case, the confidence in the estimates becomes crucial for decision making and must, thus, be quantified [Wilks, 1941; Wilks, 1942; Guba et al., 2003; Nutt et al., 2004].

This latter approach is undertaken in this work and the problem of confidence building and quantification is addressed. A sample of a small number $N$ of input parameter values 
is drawn by the Monte Carlo method from their probability distributions. The sample of $N$ input vectors thereby obtained is input to the code which is correspondingly run $N$ times, thus producing a random sample of $N$ output vectors. These can be used to estimate a given percentile of the safety margin probability distribution. To obtain the desired confidence in the safety margin percentile, the number $N$ of code runs is defined on the basis of the Order Statistics (OS) methodology, in its nonparametric formulation which applies independently from the type of probability distribution of the output data under study (in this case unknown) [Wilks, 1941; Wilks, 1942]. As we shall see, this amounts to ordering the elements of the random sample by increasing value, the element in the $r^{\text {th }}$ place being the statistic of order $r$, and using the order statistics for estimating the percentiles of the distribution (Section 2.4) with the desired confidence. Following this methodology, the number of runs required can be kept low because only statistical intervals are estimated and not the full probability distributions of the output.

\subsection{Estimation of percentiles using Order Statistics}

For ease of illustration, let us refer the discussion to a one dimensional output $y$, e.g. the Pellet Cladding Temperature (PCT) or the cooling water outlet temperature.

The $N$ runs of the code, each one with a different input vector $\bar{x}_{i}$, produce $N$ output vectors $\bar{y}_{i}, i=1,2, \ldots, N$. Let $Y=\left\{y_{1}, y_{2}, \ldots, y_{N}\right\}$ be the ordered set of values resulting from running the code $N$ times for $N$ different input vectors $X=\left\{x_{1}, x_{2}, \ldots, x_{N}\right\}$. If the code were run a very large number of times $(N \rightarrow \infty)$, it could be possible to give a sufficiently accurate estimate of the full distribution of the output $y$ and draw 
probabilistic conclusions on where it lies with respect to the threshold value $U$ (or $L$ ) defining the safety limit for the integrity of the protective barriers. This would provide a more realistic assessment than the verification that a single run conservative estimate of $y$ is within the safety envelope (e.g., PCT is less than $1200^{\circ} \mathrm{C}$ or the cooling water outlet temperature is less than $95^{\circ} \mathrm{C}$ ).

Given the computational costs associated with the estimation of the full distribution, one is forced to focus on verifying that with some level of confidence $\beta$, a certain percentage $\gamma$ of the calculated values of $y$ that would be obtained from running the code falls within the safety envelope. The thresholds defining the safety envelope and the values of the confidence and percentage are set by the regulations, considering the risk associated with exceeding the specified range.

With reference to the safety parameter $y$ to be limited from above by $U$, the approach aims at showing that the $m^{\text {th }}$ member $y_{m}$ of the $N$ sorted outputs has a certain probability $\beta$ of exceeding the unknown true $\gamma^{\text {th }}$ percentile ${ }_{\gamma} y$ [Wald, 1943; Nutt et al., 2004]. Then, one has a level of confidence $\beta$ that the actual value of ${ }_{\gamma} y$ is less than the value obtained for $y_{m}$ : if $y_{m}$ meets the criterion of being less than the safety threshold $U$, then the unknown ${ }_{\gamma} y$ will do so, too. In other words, the probabilities $\gamma$ and $\beta$ are defined as $\gamma=P\left\{y<_{\gamma} y\right\}$ and $\beta=P\left\{{ }_{\gamma} y<{ }_{\gamma} \hat{y}\right\}$.

Once $\gamma$ and $\beta$ are fixed, the OS method for calculating the $(\beta \mid \gamma)$-percentile estimate follows the lines of [Guba et al., 2003; Nutt et al., 2004] and consists in: 
i) Determining the sample size $N$ by fixing a positive integer $m$. The probability that at least $m$ observations within a random sample of size $N$ are greater than the $\gamma$-percentile of the distribution generating the sample is

$$
p=\sum_{k=0}^{N-m}\left(\begin{array}{l}
N \\
k
\end{array}\right) \gamma^{k}(1-\gamma)^{N-k}
$$

Set $p=\beta$ and compute the sample size $N$ by solving the previous equation in terms of $N$.

ii) Sorting the observations in the sample by increasing value, the element in the $r^{\text {th }}$ place being the statistic of order $r$.

iii) Estimating the $\gamma$-percentile by setting ${ }_{\gamma} \hat{y}$ equal to the statistic of order $N$ $m+1$, i.e. the $m^{\text {th }}$ largest observation in the sample; then $\beta=P\left\{{ }_{\gamma} y{ }_{\gamma} \hat{y}\right\}$. Note that higher values of $m$ in step (i) imply higher values of the sample size $N$ but generate less conservative estimates of the $\gamma$-percentile; in any case, the sample size $N$, i.e. the number of BE code runs, can be kept low because only intervals related to the $\gamma^{\text {th }}$ percentile are estimated and not the full probability distribution generating the data.

For the application of interest in the present work, the confidence intervals for the $\gamma^{\text {th }}$ percentile are computed using the $\gamma^{\text {th }}$ percentile estimates obtained from $G$ batches of newly simulated accident transients. To verify that the estimated confidence interval does not exceed the safety thresholds, two-sided confidence intervals are computed. 


\subsection{The computational approach for percentile point- and confidence intervals- estimation}

The computational approach of Figure 1 for the evaluation of a point estimate of the $\gamma$ percentile and of a confidence interval associated to it, to be used for the safety margin evaluation, is taken from [Secchi et al., 2008]. In what follows, the steps of the procedure are repeated for reader's convenience (reference distributions and notations are shown in Figure 2):

- Step 1: Code calculations. Given a set of $n_{p}$ independent input parameters values $\bar{x}_{i}, i=1,2, \ldots, n_{p}$ sampled from the relative probability distributions, a set of $n_{p}$ output values $y_{i}, i=1,2, \ldots, n_{p}$ are evaluated by the BE simulation code.

- Step 2: Code batch-calculations. Step 1 is repeated $G$ times, each time with a new sample of $N$ input values and resulting in a sample of size $N$ values $\hat{y}_{n}^{(g)}$, $n=1,2, \ldots, N, g=1,2, \ldots, G$.

- Step 3: OS batch-percentile estimation. For $g=1,2, \ldots, G$, the output sample of size $N$ of the $g^{\text {th }}$ batch is used to compute the $(\beta \mid \gamma)$-percentile estimate ${ }_{\gamma} \hat{y}^{(g)}$ by means of the OS method.

- Step 4: OS percentile estimation. The median ${ }_{\gamma} \hat{y}_{0.5}$ of the sample of the $G$ $(\beta \mid \gamma)$-percentile estimates $\hat{Y}=\left\{{ }_{\gamma} \hat{y}^{(1)},{ }_{\gamma} \hat{y}^{(2)}, \ldots,{ }_{\gamma} \hat{y}^{(G)}\right\}$ is an estimate of the median of the distribution of the estimator ${ }_{\gamma} \hat{y}$ and is used as point estimate of the $\gamma$ percentile of the output distribution for safety margin evaluations. 
- Step 5: Confidence interval calculation. In alternative to the point estimate of Step 4, we generate a confidence interval estimate of the median of the distribution of the estimator ${ }_{\gamma} \hat{y}$, as follows: $\left.i\right)$ sort $\hat{Y}=\left\{{ }_{\gamma} \hat{y}^{(1)},{ }_{\gamma} \hat{y}^{(2)}, \ldots,{ }_{\gamma} \hat{y}^{(G)}\right\}$ by increasing values and let ${ }_{\gamma} \hat{y}^{[1]},{ }_{\gamma} \hat{y}^{[2]}, \ldots,{ }_{\gamma} \hat{y}^{[G]}$ be the values of the order statistics; ii) set $r$ and $s$ to positive integers satisfying the inequality $0<r<(N+1) / 2<s \leq N$; iii) then, the random interval $\left[{ }_{\gamma} \hat{y}^{[r]}, \hat{y}^{[s]}\right]$ covers the median of the distribution of the estimator ${ }_{\gamma} \hat{y}$ with probability

$$
\alpha=I(1 / 2, N-s+1, s)-I(1 / 2, N-r+1, r)
$$

where $I(c, j, k)$ is the Regularized Incomplete Beta Function for non-singular cases [Kendall et al., 1979; Pál et al., 2002].

Hence, by fixing $\alpha$ we may find suitable $r$ and $s$, for instance in a symmetric position with respect to $(N+1) / 2$, such that $\left[{ }_{\gamma} \hat{y}^{[r]},{ }_{\gamma} \hat{y}^{[s]}\right]$ is a level- $\alpha$ confidence interval of the median of the distribution of ${ }_{\gamma} \hat{y}$.

Note that, given the fact that ${ }_{\gamma} \hat{y}$ is the $(\beta \mid \gamma)$ - estimator of the $\gamma$-percentile of the output distribution, we expect both the point estimate of Step 4 and the interval estimate of Step 5 to cover values larger than the true value of ${ }_{\gamma} y$ (see Figure 2 for an illustration of the analysis setting). The method is efficient if these estimates will not be too conservative while guaranteeing the required level of confidence. 


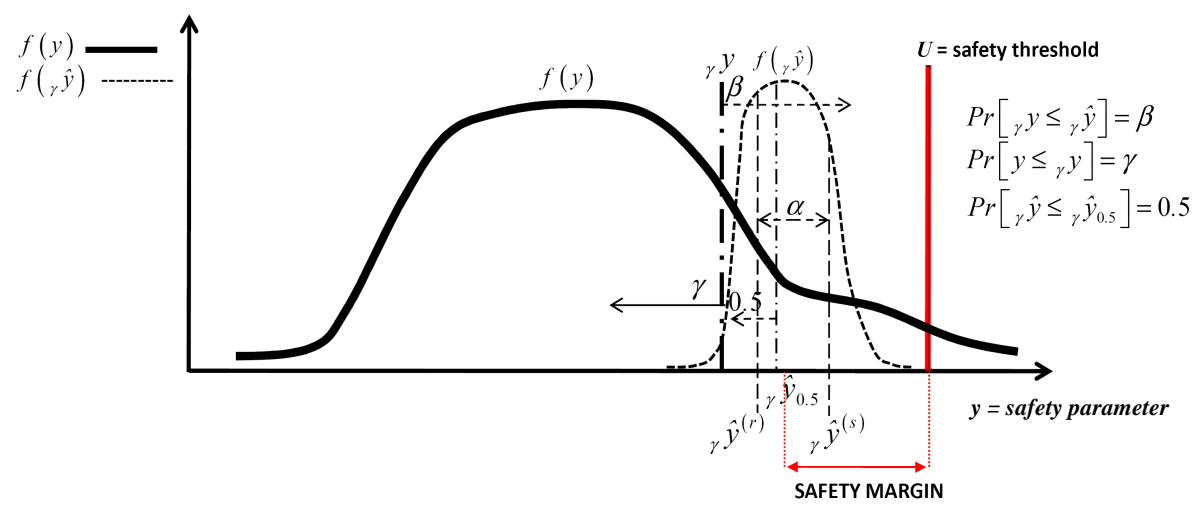

Figure 2 Sketch of the coverage value $\gamma$, the confidence $\beta$ and the confidence interval level $\alpha$; representation of the (unknown) safety parameter probability distribution $f(y)$ and its $\gamma^{\text {th }}$ percentile probability distribution $f\left({ }_{\gamma} \hat{y}\right)$

\section{THE RESIDUAL HEAT REMOVAL SYSTEM BLOCKAGE SCENARIO IN THE HTR-PM}

\subsection{The HTR-PM}

Starting from the gas-cooled reactors in the 1950s and advanced gas-cooled reactors in the 1960s, the high-temperature gas-cooled reactors have developed for nearly 50 years. Today's Chinese design of the High Temperature Gas-Cooled Reactor-Pebble bed Modular (HTR-PM) is based on the technology and experiences of the HTR-10 10MW high-temperature gas-cooled test reactor (HTR-10) designed in China in 2000.

At a first glance, the HTR-PM design has the following key technical features [Zhengy et al., 2008]:

- Characteristic coated fuel particles are used, which consist of uranium dioxide $\left(\mathrm{UO}_{2}\right)$ fuel kernel coated by tri-isotropic (TRISO) ceramics such as pyrolytic 
carbon and silicon carbide ( $\mathrm{SiC}$ ), in order to retain fission products in the particle under a fuel cladding temperature of $1600^{\circ} \mathrm{C}$ in accident cases.

- A one-zone core design is implemented, consisting of approximately 420,000 spherical fuel elements in a pebble-bed with a diameter of $3 \mathrm{~m}$ and an average height of $11 \mathrm{~m}$.

- Ceramic materials of graphite and carbon bricks, which are high-temperature resistant, surround the active reactor core.

- Decay heat in the fuel elements is dissipated by means of heat conduction and radiation to the outside of the reactor pressure vessel, and then taken away to the ultimate heat sink by water cooling panels on the surface of the primary concrete cell. Therefore, no coolant flow through the reactor core is necessary for decay heat removal in case of loss of coolant flow or loss of pressure accidents. Maximum accident fuel temperature shall be limited to $1600^{\circ} \mathrm{C}$.

- Spherical fuel elements are charged and discharged in a so-called "multi-pass" mode, which means that before the fuel elements reach the discharge burn-up, they go through the reactor core several times.

- Several of HTR-PM modular reactors can be built at one site to satisfy the power capacity demand of the utility. Some auxiliary systems and facilities can be shared among the modules. 


\subsection{The Passive RHRs}

The enhanced safety of the HTR-PM is mainly due to the implementation of passive safety systems [Zhao et al., 2008].

Figure 3 sketches the equipment layout of one of the 3 loops of the RHR system implemented in the HTR-PM. The water cooled wall gets the heat from the reactor vessel by thermal radiation; then, the pipe transfers the water to the air-cooled heat exchanger located in the air-cooled tower; the cool air takes the heat away from the heat exchanger to the environment.

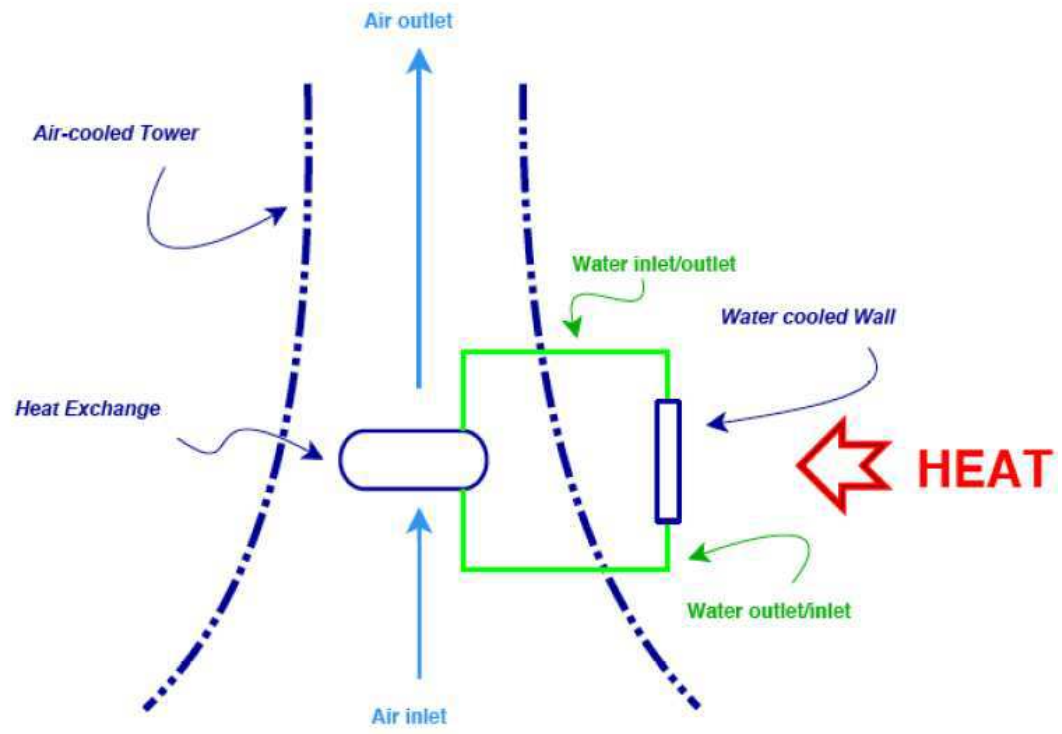

Figure 3 Schematics of 1 loop of the RHRs in the HTR-PM [Zhao et al., 2008]

\subsection{The RHRs blockage scenarios}

The outlet water temperature $T_{w, \text { out }}$ of the RHRs is considered as the safety parameter with respect to which the success or failure of the system is defined. From the 
engineering experience, when $T_{w, \text { out }}$ exceeds the critical temperature $T_{c}$, local boiling may occur which can significantly worsen thermal transmission. Although engineering experience recommends $T_{c}=95^{\circ} \mathrm{C}$, in this work, a value of $T_{c}=90^{\circ} \mathrm{C}$ was conservatively chosen.

The accident scenarios considered in the present study are:

- Scenario A: 2/3 RHR loops out of service (i.e., simultaneously failed and/or under maintenance: this constitutes a BDBA)

- Scenario B: 1/3 RHR loops out of service (i.e., failed or under maintenance: this is a scenario included in the set of DBA)

- Scenario C: 0/3 RHR loops out of service (i.e., nominal condition)

Scenarios A and B lead to a temporary decrease in the cooling capability of the RHR and to a corresponding increase of $T_{w, \text { out }}$ which may exceed $T_{c}$ [Zhao et al., 2008]. For this reason, these scenarios are considered safety relevant and a careful analysis of it must be performed.

\subsection{Simulations of the accident scenarios}

A simplified zero-dimensional description of the thermo-hydraulic behavior of the RHRs has been implemented in MATLAB and used to simulate accident blockage transients. The model allows the computation of the maximum outlet water temperature reached during an accident scenario.

The simulation code models the following phases of the process: 
1. The residual heat radiates from the reactor vessel and other thermal sources to the water in the water-cooled wall;

2. Because of the difference in temperature, natural convection will initiate through water, in the water-cooled wall and pipes connected with the air-cooled heat exchanger; then, heat will transfer to the water side of the heat exchanger;

3. The heat will transfer by thermal conduction from the water side to the air side of the heat exchanger, due to the difference of temperature;

4. As the air-cooled heat exchanger is located in the air-cooled tower, natural convection of air will set up and take heat to the final heat trap-atmosphere.

The model fed with the nominal "best estimate" values of the input parameters is assumed to be "sufficiently best estimate". The RHRs accident complete blockage transients are generated by sampling the involved 37 input parameters from probability distributions defined on the basis of previous experience and/or information obtained by skilled experts (Table 1).

\begin{tabular}{|c|c|c|l|}
\hline $\boldsymbol{N}$ & Parameter & Distribution & Note \\
\hline 1 & $W$ & Normal & Residual heat power \\
\hline 2 & $T_{a, \text { in }}$ & Bi-Normal & Temperature of inlet air in the air cooled tower \\
\hline 3 & $x_{i 1}$ & Uniform & Resistance coefficient of elbow \\
\hline 4 & $x_{i 2}$ & Uniform & Resistance coefficient of header channel \\
\hline 5 & $x_{i w}$ & Uniform & Resistance coefficient of the water tank walls \\
\hline 6 & $x_{i a, \text { in }}$ & Uniform & Sum of the resistance coefficients of inlet shutter and air cooling tower and silk net \\
\hline 7 & $x_{i a, \text { out }}$ & Uniform & Sum of the resistance coefficients of outlet shutter and air cooling tower and silk net \\
\hline 8 & $x_{i a, \text { arrow }}$ & Uniform & Resistance coefficient of the narrowest part of the tower \\
\hline 9 & $P_{a, \text { in }}$ & Uniform & Pressure of the inlet air in the cooler tower \\
\hline 10 & $d_{x}$ & Uniform & Roughness of pipes \\
\hline 11 & $H_{a}$ & Normal & Height of chimney \\
\hline 12 & $L_{a}$ & Normal & Length of pipes in the exchanger \\
\hline 13 & $N_{a}$ & Normal & Total number of pipes in the air cooler \\
\hline 14 & $A_{f}$ & Normal & Air flow crossing are in the narrowest part of the tower \\
\hline 15 & $A_{f, \text { in }}$ & Normal & Inlet air flow crossing area in the tower \\
\hline 16 & $A_{f, o u t}$ & Normal & Outlet air flow crossing area from the tower \\
\hline 17 & $A_{f, \text { narrow }}$ & Normal & Crossing area in the narrowest part of the tower \\
\hline 18 & $S_{1}$ & Normal & Distance between centers of adjacent pipes in horizontal direction \\
\hline 19 & $S_{2}$ & Normal & Distance between centers of adjacent pipes in vertical direction \\
\hline 20 & $S$ & Normal & Distance between fins in the ribbed pipe \\
\hline 21 & $D_{a}$ & Normal & Pipes inner diameter in the air cooling exchanger \\
\hline 22 & $D_{o}$ & Normal & Pipes outer diameter \\
\hline
\end{tabular}




\begin{tabular}{|c|c|c|l|}
\hline 23 & $D_{\text {outer }}$ & Normal & Rib outer diameter \\
\hline 24 & $P_{w}$ & Normal & Water pressure in the pipes \\
\hline 25 & $H_{w}$ & Normal & Elevatory height of water \\
\hline 26 & $N_{w}$ & Discrete Normal & Number of water cooling pipes for each loop \\
\hline 27 & $L_{w}$ & Normal & Length of the water cooling pipes \\
\hline 28 & $D_{w}$ & Normal & Inner diameter of the water cooling pipes \\
\hline 29 & $D_{l}$ & Normal & Inner diameter of the in-core and air cooler connecting pipes \\
\hline 30 & $D_{2}$ & Normal & Inner diameter of the in-core header \\
\hline 31 & $L_{C}$ & Normal & Length of the in-core and air cooler connecting pipes ("cold leg") \\
\hline 32 & $L_{H}$ & Normal & Length of the in-core and air cooler connecting pipes ("hot leg") \\
\hline 33 & $R_{i}$ & Log-normal & Thermal resistance of pipes inside of the heat exchanger \\
\hline 34 & $R_{o}$ & Log-normal & Thermal resistance due to the dirt of the pipes fins \\
\hline 35 & $R_{g}$ & Log-normal & Thermal resistance of the gap between fins \\
\hline 36 & $R_{f}$ & Log-normal & Thermal resistance of fins \\
\hline 37 & lamd & Normal & Heat transfer coefficient of the pipes \\
\hline
\end{tabular}

Table 1 Parameters which are regarded relevant for the behavior of the passive RHRs.

\section{RESULTS}

The non-parametric procedure for percentile estimation introduced in Section 2.5 is hereafter illustrated with reference to the estimation of the safety margin of the maximum outlet water temperature $T_{w, \text { out }}$ reached during the accident scenarios $\mathrm{A}, \mathrm{B}$ and $\mathrm{C}$ of complete/partial blockage of the passive RHRs of the HTR-PM described in Section 3.

Order Statistics has been applied to a sample of maximum outlet water temperature values obtained by simulation, for estimating the $\gamma^{\text {th }}$ percentile with $\gamma=0.95$. Then, $G$ estimates of the $\gamma^{\text {th }}$ percentile have been collected by the OS applied to $G$ different batch samples. Finally, confidence intervals for the real $\gamma^{\text {th }}$ percentile have been evaluated.

The procedural steps described in Section 2.5 have been performed as follows:

\section{Step 1: BE code calculations.}

To demonstrate the feasibility of the procedure, we take $m=1$ and $\beta=\gamma=0.95$; this leads to the smallest sample size $N=59$ for the OS $(\beta \mid \gamma)$-percentile estimates.

\section{Step 2: Code batch-calculations.}

A number of $G=50$ batches of $N$ output values have been computed. 


\section{Step 3: OS batch-percentile estimation.}

For each of the $G=50$ batches, the $(\beta \mid \gamma)$-percentile estimates have been computed and collected in the sample $\hat{Y}=\left\{{ }_{0.95} \hat{y}^{(1)},{ }_{0.95} \hat{y}^{(2)}, \ldots,{ }_{0.95} \hat{y}^{(50)}\right\}$.

\section{Step 4: OS percentile estimation.}

The median of the sample $\hat{Y}$ and its safety margin are evaluated for the accident scenarios A, B and C. The results are provided in the second column of Tables 2, 3 and 4, respectively.

\section{Step 5: Confidence interval calculation.}

Because of the limitation on the sample size used in the estimation, the safety acceptance criteria cannot be based solely on the best estimate results. Hence, the uncertainty of the estimated safety margin must be properly informed, e.g. by computing its confidence interval. With reference to the accident scenarios $\mathrm{A}, \mathrm{B}$ and $\mathrm{C}$, the confidence interval of level $\alpha=0.95$, with $r=1$ and $s=49$, are provided in the second column of Tables 2, 3 and 4, respectively.

\begin{tabular}{|c|c|c|}
\hline \multicolumn{2}{|c|}{ Scenario A } \\
\hline $\boldsymbol{m}$ & \multicolumn{2}{|c|}{1} \\
\hline $\boldsymbol{N}$ & 50 & 100 \\
\hline $\boldsymbol{G}$ & -6.67 & -6.55 \\
\hline Median safety margin ${ }_{0.95} \hat{y}_{0.5}(A)$ & {$[-3.12,-11.85]$} & {$[-3.07,-11.38]$} \\
\hline Confidence interval $\left[{ }_{0.95} \hat{y}^{(3)}(A),{ }_{0.95} \hat{y}^{(97)}(A)\right]$ &
\end{tabular}

Table 2 Median of the $95^{\text {th }}$ percentile distribution and the corresponding confidence interval, obtained for the accident scenario $\mathrm{A}, m=1, N=50$ and $G=50,100$. 


\begin{tabular}{|c|c|c|}
\hline \multicolumn{3}{|c|}{ Scenario B } \\
\hline $\boldsymbol{m}$ & \multicolumn{2}{|c|}{1} \\
\hline $\boldsymbol{N}$ & 50 & 100 \\
\hline $\boldsymbol{G}$ & 37.78 & 37.29 \\
\hline Median safety margin ${ }_{\gamma} \hat{y}_{0.5}(B)$ & {$[40.72,34.01]$} & {$[40.88,33.19]$} \\
\hline Confidence interval $\left[{ }_{0.95} \hat{y}^{(3)}(B),{ }_{0.95} \hat{y}^{(97)}(B)\right]$ & \multirow{2}{*}{\begin{tabular}{c}
$|c|$ \\
\hline
\end{tabular}}
\end{tabular}

Table 3 Median of the $95^{\text {th }}$ percentile distribution and the corresponding confidence interval, obtained for the accident scenario $\mathrm{B}, m=1, N=50$ and $G=50,100$.

\begin{tabular}{|c|c|c|}
\hline \multicolumn{2}{|c|}{ Scenario C } \\
\hline $\boldsymbol{m}$ & \multicolumn{2}{|c|}{1} \\
\hline $\boldsymbol{N}$ & 50 & 100 \\
\hline Median safety margin ${ }_{0.95} \hat{y}_{0.5}(C)$ & 56.49 & 56.55 \\
\hline Confidence interval $\left[{ }_{0.95} \hat{y}^{(3)}(C){ }_{0.95} \hat{y}^{(97)}(C)\right]$ & {$[58.92,52.59]$} & {$[58.79,52.70]$} \\
\hline
\end{tabular}

Table 4 Median of the $95^{\text {th }}$ percentile distribution and the corresponding confidence interval, obtained for the accident scenario $\mathrm{C}, m=1, N=50$ and $G=50,100$.

It can be seen that:

- scenario A has a negative safety margin: the occurrence of this accident scenario has to be avoided;

- from scenario C to scenario B the safety margin shrinks;

- scenarios B and C can be classified as safe, because with positive safety margins. This means that, in absence of any other component failure in the system, although one of the loops is failed the system can continue to produce energy; on the other hand, in case of occurrence of scenario B, a maintenance action has to be adopted quickly to reactivate the failed RHR loop;

- as shown in Figure 4, in general terms, the probability distributions of the safety parameter $y \quad f(y \mid$ scenario $\mathrm{X})$, with $\mathrm{X}=\mathrm{A}, \mathrm{B}$ and $\mathrm{C}$, are different. As a consequence, also the distributions of the 95-th percentiles $f\left({ }_{0.95} \hat{y} \mid\right.$ scenario $\left.\mathrm{X}\right)$, based on the results obtained with the $G$ estimates of ${ }_{0.95} \hat{y}$, are different: the more 
the accident scenario is unsafe, the more the related distributions shift towards the safety threshold (Figure 4).

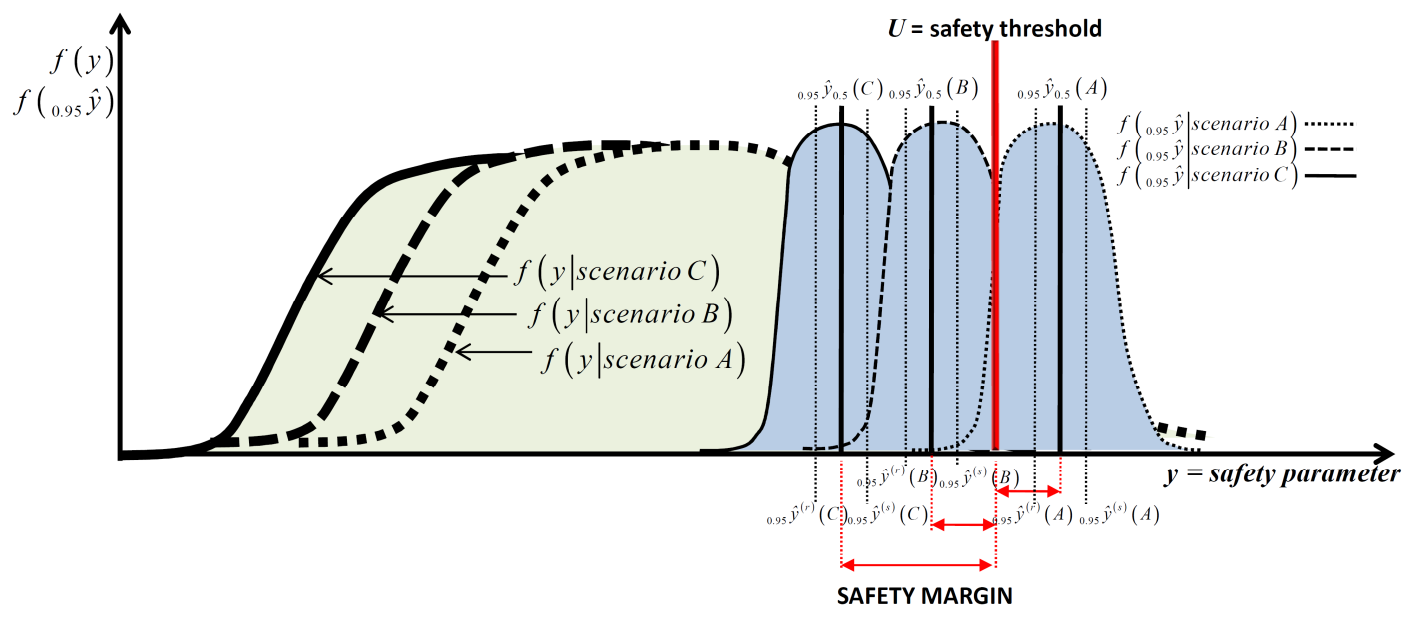

Figure 4 Conceptual sketch of the results obtained for the accident scenarios A, B and C

\subsection{Improvements of the estimation accuracy by means of higher values of $m, N$ and $G$}

It is known that using higher values of $m, N$ and $G$ would allow increasing the reliability on the estimated confidence interval [Zio et al., 2008]. Indeed, conservatism is reduced by taking higher values of $m, N$ and $G$ [Nutt et al., 2004] and, by comparison of the results, the analyst would feel reassured that the estimates obtained have a low probability of differing significantly from the true values (usually unknown), and that the estimated maximum outlet water temperature value satisfies the safety threshold limit $U$. In this view, the same procedure detailed in Section 2.5 has been repeated increasing the number of samples $G$. For the accident scenarios A, B and C, the results are provided 
with $m=1, N=59, G=100, \alpha=0.95, r=3$ and $s=97$ and reported in the third column of Tables 2, 3 and 4, respectively:

- despite the higher computational time $\theta \propto b(N \cdot G)$, with $b$ a constant coefficient, and the greater accuracy in the estimates, the medians for the 3 accident scenarios are practically the same.

- for all 3 accident scenarios, the application of the procedure with $G=100$ reduces the conservatism in the results since it provides the lowest point-estimate values of the maximum outlet water temperature (larger positive safety margins for scenarios B and C and smaller negative safety margin for Scenario A).

For comparison, the procedure detailed in Section 2.5 has been repeated increasing the number of simulations $N$ : the value $m=50$ leads to a sample size $N=1228$. The results are provided in Tables 5,6 and 7. It can be seen that:

- increasing $m$ and correspondingly increasing the number of values that are requested at least to lie beyond the "extent" $\gamma$ of the cumulative probability, the estimation ${ }_{0.95} \hat{y}$ of the percentile tends to narrow the true ${ }_{0.95} y$, which have been evaluated running the code 100000 times for each accident scenario. By testing the 100000 outputs for Normality by means of the Lilliefors Test [Lilliefors, 1967] and then applying a parametric approach for the 95-th percentile estimations, the estimate of ${ }_{0.95} y$ has turned out to be equal to $-3.22,40.66$ and 58.70 for scenarios A, B and C, respectively. 
- for all the 3 accident scenarios the application of the procedure with $m=50$ increases the confidence on the estimated percentile value with respect to that with $m=1$, as demonstrated by the shrinking of the confidence intervals.

As an example, Figure 5 shows the combined effect of larger values of $m$ and $G$ with reference to the results provided for the accident scenario $\mathrm{C}$.

\begin{tabular}{|c|c|c|}
\hline \multicolumn{2}{|c|}{ Scenario A } \\
\hline $\boldsymbol{m}$ & \multicolumn{2}{c|}{50} \\
\hline $\boldsymbol{N}$ & 100 & 1228 \\
\hline $\boldsymbol{G}$ & -3.86 & -3.93 \\
\hline Median safety margin ${ }_{0.95} \hat{y}_{0.5}(A)$ & {$[-3.76,-4.24]$} & {$[-3.23,-4.63]$} \\
\hline Confidence interval $\left[{ }_{0.95} \hat{y}^{(4)}(A){ }_{0.95} \hat{y}^{(147)}(A)\right]$ &
\end{tabular}

Table 5 Median of the $95^{\text {th }}$ percentile distribution and the corresponding confidence interval, obtained for the accident scenario $A, m=50, N=1280$ and $G=100,150$.

\begin{tabular}{|c|c|c|}
\hline \multicolumn{2}{|c|}{ Scenario B } \\
\hline $\boldsymbol{m}$ & \multicolumn{2}{|c|}{50} \\
\hline $\boldsymbol{N}$ & 100 & 1228 \\
\hline $\boldsymbol{G}$ & 39.86 & 39.85 \\
\hline Median safety margin ${ }_{0.95} \hat{y}_{0.5}(B)$ & {$[40.53,39.22]$} & {$[40.56,39.12]$} \\
\hline Confidence interval $\left[{ }_{0.95} \hat{y}^{(4)}(B){ }_{0.95} \hat{y}^{(147)}(B)\right]$ & \multirow{2}{*}{\begin{tabular}{c}
$|c|$ \\
\hline
\end{tabular}}
\end{tabular}

Table 6 Median of the $95^{\text {th }}$ percentile distribution and the corresponding confidence interval, obtained for the accident scenario $B, m=50, N=1280$ and $G=100,150$.

\begin{tabular}{|c|c|c|}
\hline \multicolumn{3}{|c|}{ Scenario C } \\
\hline $\boldsymbol{m}$ & \multicolumn{2}{|c|}{50} \\
\hline $\boldsymbol{N}$ & 100 & 1228 \\
\hline $\boldsymbol{G}$ & 58.27 & 58.28 \\
\hline Median safety margin ${ }_{0.95} \hat{y}_{0.5}(C)$ & {$[58.82,57.71]$} & {$[58.83,57.78]$} \\
\hline Confidence interval $\left[{ }_{0.95} \hat{y}^{(4)}(C),{ }_{0.95} \hat{y}^{(147)}(C)\right]$ &
\end{tabular}

Table 7 Median of the $95^{\text {th }}$ percentile distribution and the corresponding confidence interval, obtained for the accident scenario $C, m=50, N=1280$ and $G=100,150$. 


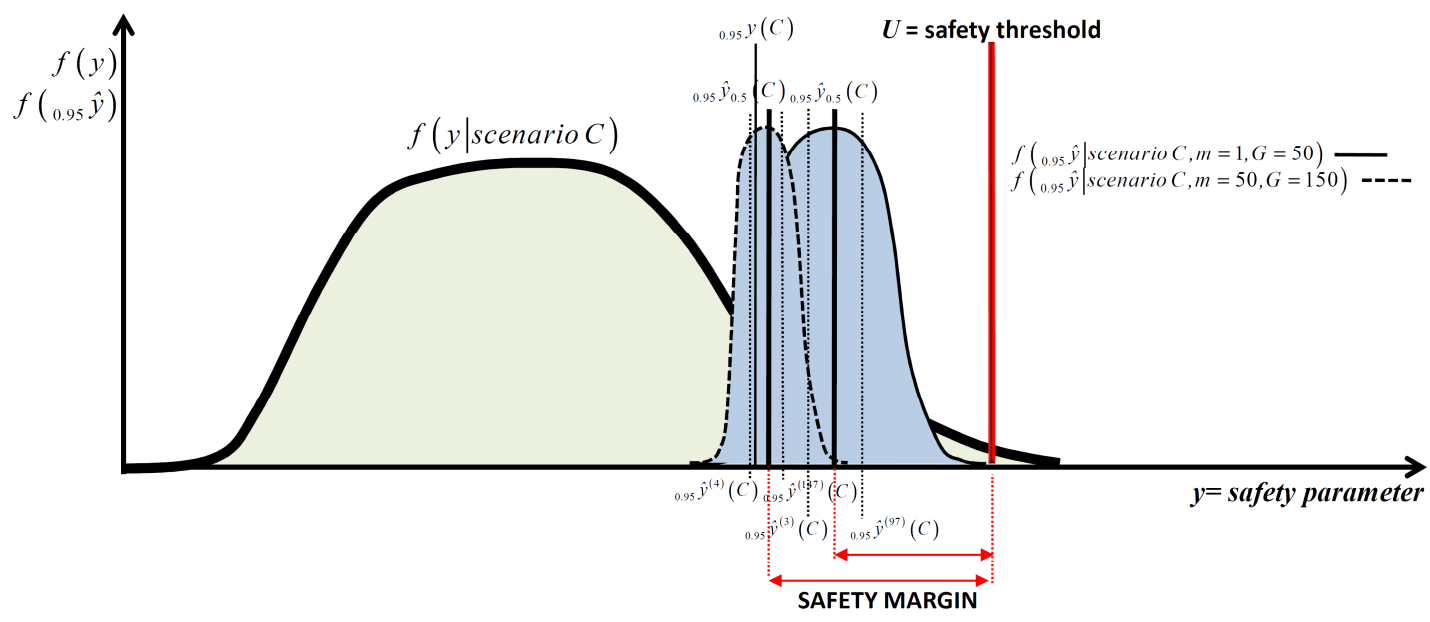

Figure 5 Conceptual comparison of the results obtained for the accident scenario $\mathrm{C}$ with $m=1,50$ and $G=50,150$, respectively

\subsection{Safety margin estimation based on a reduced set of input parameters}

The same calculations have been repeated by sampling values only of the power $W$, the inlet temperature of air in the air-cooled tower $T_{a, \text { in }}$ and the water pressure in the pipes $P_{w}$, which have been identified by sensitivity analysis as the most relevant parameters affecting the outlet water temperature $T_{w, \text { out }}$ [Yu et al, 2010a; Yu et al., 2010b]. The results are provided in Tables 8-10. By comparison to Tables 2-4, respectively, it can be seen that an overall qualitative agreement exists between the safety margins evaluated resorting to the complete analysis considering all 37 input parameters and those obtained with only the 3 most relevant input parameters: this demonstrates the efficacy of sensitivity analysis and allows for an even faster safety margin evaluation, freeing the procedure from the numerous time-consuming samplings of all the input parameters values and related code calculations. 


\begin{tabular}{|c|c|c|}
\hline \multicolumn{3}{|c|}{ Scenario A } \\
\hline $\boldsymbol{m}$ & \multicolumn{2}{|c|}{1} \\
\hline $\boldsymbol{N}$ & 50 & 100 \\
\hline $\boldsymbol{G}$ & -7.51 & -7.08 \\
\hline Median safety margin ${ }_{0.95} \hat{y}_{0.5}(A)$ & {$[-3.07,-11.13]$} & {$[-3.49,-11.36]$} \\
\hline Confidence interval $\left.{ }_{0.95} \hat{y}^{(3)}(A){ }_{0.95} \hat{y}^{(97)}(A)\right]$ &
\end{tabular}

Table 8 Median of the $95^{\text {th }}$ percentile distribution and the corresponding confidence interval, obtained for the accident scenario $A, m=1, N=50, G=50,100$ and only 3 input parameters.

\begin{tabular}{|c|c|c|}
\hline \multicolumn{3}{|c|}{ Scenario B } \\
\hline $\boldsymbol{m}$ & \multicolumn{2}{c|}{1} \\
\hline $\boldsymbol{N}$ & 50 & 100 \\
\hline $\boldsymbol{G}$ & 37.56 & 37.34 \\
\hline Median safety margin ${ }_{0.95} \hat{y}_{0.5}(B)$ & {$[41.06,33.14]$} & {$[40.83,33.19]$} \\
\hline Confidence interval $\left[{ }_{0.95} \hat{y}^{(3)}(B){ }_{0.95} \hat{y}^{(97)}(B)\right]$ & \multicolumn{2}{|c|}{} \\
\hline
\end{tabular}

Table 9 Median of the $95^{\text {th }}$ percentile distribution and the corresponding confidence interval, obtained for the accident scenario $B, m=1, N=50, G=50,100$ and only 3 input parameters.

\begin{tabular}{|c|c|c|}
\hline \multicolumn{3}{|c|}{ Scenario C } \\
\hline $\boldsymbol{M}$ & \multicolumn{2}{|c|}{1} \\
\hline $\boldsymbol{G}$ & 50 & 100 \\
\hline Median safety margin ${ }_{0.95} \hat{y}_{0.5}(C)$ & 55.65 & 56.31 \\
\hline Confidence interval $\left[{ }_{0.95} \hat{y}^{(3)}(C){ }_{0.95} \hat{y}^{(97)}(C)\right]$ & {$[58.88,53.36]$} & {$[58.73,52.59]$} \\
\hline
\end{tabular}

Table 10 Median of the $95^{\text {th }}$ percentile distribution and the corresponding confidence interval, obtained for the accident scenario $C, m=1, N=50, G=50,100$ and only 3 input parameters.

Finally, the physical conclusion that can be drawn from the analysis is that, for the safe operation of the plant, two RHR loops are enough; the $3^{\text {rd }}$ loop can be considered as a redundancy in the RHRs to guarantee high availability of the safety function. The large safety margins computed in case of the safe scenarios (B and C) suggest a possible improvement of the whole system design to avoid excessive conservatism leading to a more efficient plant design and operation. 


\section{CONCLUSIONS}

A computational framework of literature has been applied for the estimation of the safety margin on the maximum outlet water temperature of the passive RHRs reached during some accident scenarios of the HTR-PM.

The procedure exploits non-parametric Order Statistics performed on a limited number of BE code calculations for providing confidence intervals on the estimated percentiles. An analysis has been performed on the effects of some key parameters, related to the size of the statistical sample and on the number of uncertain input variables considered in the analysis.

The procedure has been demonstrated to give reliable (the estimates are similar despite of the increase of the number of simulation), robust (confidence intervals are very narrow) and conservative (increasing the number of simulations, the estimates tend to narrow down to the true value) estimates of the $95^{\text {th }}$ percentiles of the safety parameters distributions.

The method has been demonstrated effective in that it is capable of indicating the passive system safety conditions, accounting for the uncertainties in the model parameters and in the estimate itself.

\section{ACKOWLEDGEMENTS}

This work was supported in part by the Science \& Technology Fellowship Programme in China financed by the European Commission (EuropeAid/127024/L/ACT/CN) and by

the Important National Science \& Technology Specific Projects in China (No. 2008ZX06902-009). 


\section{REFERENCES}

[Apostolakis, 1990] Apostolakis, G.E., The Concept of Probability in Safety Assessments of Technological Systems, Science, pp. 1359-1364, 1990.

[Burgazzi, 2007] Burgazzi, L., Thermal-hydraulic passive system reliability based design approach, Reliability Engineering and System Safety, doi:10.1016/j.ress.2006.07.008, 2007.

[Gavrilas et al., 2004] Gavrilas, M.,Meyer, j. Youngblood, B., Prelewicz, D., Beaton, R., A Generalized Framework for Assessment of Safety Margins in Nuclear Power Plants, Proceedings to BE 2004: International Meeting on Updates in Best Estimate Methods in Nuclear Installations Safety Analysis, Washington, DC, November 14-18, 2004.

[Guba et al., 2003] Guba, A, Makai, M, Pa'1, L., Statistical Aspects of Best Estimate Method-1, Reliability Engineering and System Safety, 80, 217-32, 2003.

[IAEA, 1991] Safety related terms for advanced nuclear plant. IAEA TECDOC-626.

[Helton, 2004] Helton, J.C., Alternative Representations of Epistemic Uncertainty, Special Issue of Reliability Engineering and System Safety, Vol. 85, 2004.

[Lilliefors, 1967] Lilliefors, H., On the Kolmogorov-Smirnov test for normality with mean and variance unknown", Journal of the American Statistical Association, Vol. 62. pp. 399-402, 1967.

[Marquès et al., 2005] Marquès, M., Pignatel, J.F., Saignes, P., D’Auria, F., Burgazzi, L., Müller, C., Bolado-Lavin, R., Kirchsteiger, C., La Lumia, V., Ivanov, I., Methodology for the reliability evaluation of a passive system and its integration into a probabilistic safety assessment. Nuclear Engineering and Design 235, 2612-2631, 2005.

[Marseguerra et al., 2003] Marseguerra, M., Masini, R., Zio, E. and Cojazzi, G., Variance Decomposition-Based Sensitivity Analysis via Neural Networks. Reliability Engineering and System Safety, 79 , 229-238, 2003.

[Marseguerra et al., 2004] Marseguerra, M., Zio, E. and Canetta, R., Using Genetic Algorithms for Calibrating Simplified Models of Nuclear Reactor Dynamics. Annals of Nuclear Energy, 31, 1219-1250, 2004.

[Martorell et al., 2006] Martorell, S., Nebot, Y., Villanueva, J.F., Carlos, S., Serradell, V., Pelayo, F., Mendizàbal, R., Safety Margins Estimation Method Considering Uncertainties within Risk-Informed Decision Making Framework. In "Proceedings of the PHYSOR 2006 Conference", Vancouver, Canada, September 10-14, 2006.

[Mathews et al., 2008] Mathews, T.S., Ramakrishnan, M., Parthasarathy, U., John Arul, A., Senthil Kumar, C, Functional reliability analysis of safety grade decay heat removal system of Indian 500MWe PFBR. Nuclear Engineering and Design 238, 2369-2376, 2008.

[Nutt et al., 2004] Nutt, W.T, Wallis, G.B., Evaluation of Nuclear Safety from the outputs of Computer Codes in the Presence of Uncertainties, Reliability Engineering and System Safety, 83, 57-77, 2004.

[Pagani et al., 2005] Pagani, L., Apostolakis, G.E., Hejzlar, P., The impact of uncertainties on the performance of passive systems. Nuclear Technology 149, 129-140, 2005.

[Secchi et al., 2008] P. Secchi, E. Zio, F. Di Maio, Quantifying Uncertainties in the Estimation of Safety Parameters by Using Bootstrapped Artificial Neural Networks, Annals of Nuclear Energy, pp. 2338-2350(35), doi: 10.1016/j.anucene.2008.07.010, 2008.

[USNRC, 1998] USNRC, 1998, An Approach for using probabilistic risk assessment in riskinformed decisions on plant-specific changes to the licensing basis, RG 1.174 
[Wilks, 1941] Wilks, SS., Determination of Sample Sizes for Setting Tolerance Limits, Ann Math Stat;12:91-6, 1941

[Wilks, 1942] Wilks, SS., Statistical Prediction with Special Reference to the Problem of Tolerance Limits. Ann Math Stat;13:400-9, 1942.

[Wald, 1943] Wald, A., An extension of Wilks method for setting tolerance limits, Ann Math Stat; $14,45-55,1943$.

[Yu et al., 2010a] Y. Yu , T. Liu, J.Tong, J. Zhao, F. Di Maio, E. Zio, The Analytic Hierarchy Process for the Sensitivity Analysis of Passive Safety System, The 10th Conference on Probabilistic Safety Assessment and Management (PSAM10), Seattle, 7-11 June 2010.

[Yu et al., 2010b] Y. Yu , T. Liu, J.Tong, J. Zhao, F. Di Maio, E. Zio, Variance Decomposition Method for Passive System Models Sensitivity Analysis, The 10th Conference on Probabilistic Safety Assessment and Management (PSAM10), Seattle, 7-11 June 2010.]

[Zhao et al., 2008] Zhao, R., Zhao, J., Liu, T., Tong, J., Assessing Physical Process Failure Probability of Passive System in an Advanced Reactor by Using M.C. Method, Proceedings of PSAM9, Hong Kong, May 2008.

[Zio et al., 2008] Zio, E., Di Maio, F., Using Order Statistics and the Bootstrap Technique for quantifying Thermal-Hydraulic Code Uncertainties in the Estimation of Probabilistic Safety Margins, Science and Technology of Nuclear Installations, doi:10.1155/2008/340164, 2008.

[Zhengy et al., 2008] Zhengy, Y., Shi, L., Characteristics of the 250MW Pebble-Bed Modular High Temperature Gas-Cooled Reactor in Depressurized Loss of Coolant Accidents, Proceedings of the 4th International Topical Meeting on High Temperature Reactor Technology, HTR2008, Washington, DC USA, 2008. 